\title{
Responsabilité sémantique et responsabilité éthique
}

\section{Jean-Michel Salanskis}

Cette intervention rend compte d'une certaine manière de réagir à ce qui est éprouvé comme la situation contemporaine de la philosophie du langage, et ce à partir d'une sensibilité lévinasienne. Je conduirai l'exposé jusqu'au point de présenter dans ses grandes lignes la démarche philosophique qui, chez moi, se substitue pour ainsi dire aux approches de philosophie du langage aujourd'hui disponibles, démarche dont les tenants et les aboutissants furent dévoilés dans Sens et philosophie $d u$ sens. ${ }^{1}$ Mais je le ferai en rapportant en même temps le contexte où cette démarche s'inscrit, et en ayant égard à la pensée d'Emmanuel Levinas, dont j'ai dit qu'elle définissait en l'espèce ma sensibilité.

\section{Levinas et le langage}

Pour aborder mon sujet devant vous et avec vous, je commencerai par une brève évocation d'un certain nombre 
d'éléments ayant traits au langage que l'on trouve chez Emmanuel Levinas. On ne peut pas dire, en effet, que le thème du langage soit absent de sa philosophie, il intervient plutôt de manière à chaque fois décisive pour le propos lévinasien, donnant matière à des mises au point ou développements de grande profondeur et de grande portée, dont la succession jalonne son œuvre. On peut mentionner ainsi:

1. L'idée, avancée dans Totalité et infini, que le discours est pour ainsi dire adapté à la relation non thématisante à l'altérité d'autrui, puisque, allant chercher ce dernier sur le mode de l'interpellation, il manifeste sa distance "abyssale" de non-objet et la "franchit en la respectant" de la seule possible façon. Autrui est d'ailleurs respecté en ce sens, selon Levinas, même quand je le catégorise.

2. La présentation dans Autrement qu'être de l'ordre du langage en général, en tant que forme de l'universel et du rationnel, comme motivé par l'exigence, apparue avec le tiers, de comparer les demandes qui émanent des "divers visages", par l'effort pour établir quelque chose comme une peréquation des multiples dettes, toutes infinies, dont l'humanité est le tissu: le vrai monde, non limité à l'intrigue duelle, est à ce prix.

3. L'évocation, au début de L'au-delà du verset, des littératures nationales, de ces textes-récits océaniques où une famille de l'humanité puise indéfiniment de quoi confirmer et réinterpréter une identité. Mentionnant ainsi Shakespeare, Molière, Dante, Cervantès, Goethe ou Pouchkine comme les auteurs d'autant de 'Torahs non juives pour ainsi dire, Levinas insiste sur l'importance du niveau du livre, privilégiant un pallier de la manifestation linguistique au lieu de s'en tenir à son soussol technique ou grammatical.

4. Le lien établi, dans Autrement qu'être à nouveau, entre l'impossibilité de refouler définitivement le scepticisme et l'invincible récurrence du Dit en écho au Dire aussi bien que du Dire derrière tout Dit. Levinas semble toucher, cette fois, à 


\section{RESPONSABILI'IÉ SÉMANITQUE}

des réflexions relevant de ce qui s'appelle de manière contemporaine le niveau pragmatique.

En dépit de la force de l'image philosophique du langage que l'on peut constituer à partir de la juxtaposition de tels éléments, on ne peut pas dire qu'ils composent, collectivement, ce qui devrait être appelé une philosophie du langage lévinasienne. C'est que, tout simplement, dans la mise en avant de chacun d'entre eux, ce n'est pas d'abord du langage qu'il s'agit pour Levinas: plutôt, de l'interpellation pour 1), de la justice pour 2), de la littérature pour 3), et de la négativité pour 4).

Si l'on veut trouver chez Levinas une posture philosophique concernant, fût-ce indirectement, le langage comme tel, il faut, je pense, plutôt la chercher du côté de ce qu'il dit sur le sens. Et, d'ailleurs, il me semble qu'une orientation lévinasienne nous conduit à interroger les doctrines philosophiques standard traitant du sens, et les façons pour la philosophie de prendre la mesure de ou atteindre la profondeur du sens.

\section{Philosophie du langage contemporaine et question du sens}

Ce que nous avons sous les yeux, dans la philosophie du vingtième siècle, ce ne sont pas des philosophie du sens, mais plutôt des philosophies du langage. La figure de la philosophie du langage est dominatrice au sein du siècle en question, sous le double visage d'une philosophie analytique du langage issue des travaux de Frege et de Russell et d'une philosophie herméneutico-phénoménologique du langage largement inspirée de Heidegger, mais prenant aussi ses racines auprès de Husserl. 
En même temps, d'un côté comme de l'autre, on prétend à certains égards prendre en charge la question du sens, ne s'occuper que du sens même.

De Frege à Dummett, la philosophie analytique s'est constituée comme réflexion aiguë et profonde sur le langage et sur la signification linguistique, en telle sorte que les écrits qui interrogent le sens sont nombreux, et coïncident avec les maitres ouvrages du courant. Kripke se demande dans Naming and Necessity quel est au fond le sens d'un nom propre, Dummett pose, dans un célèbre double article, la question What is a Theory of Meaning?

Husserl ne cesse d'utiliser le mot sens, et s'attache à décrire le sens d'objet caractéristique de chaque "région". Heidegger pose originairement la question du "sens de l'être" et dégage l'herméneutique comme l'âme ou le mouvement propres de la pensée.

Pourquoi puis-je néanmoins me déclarer insatisfait, et continuer de chercher ce qui pourrait être une vraie et une bonne "philosophie du sens"? C'est, d'abord, pour un motif lévinasien en quelque sorte: parce que je crois reconnaitre dans l'approche de base de la philosophie analytique et dans celle de la phénoménologie, bientôt relayée par l'herméneutique, la même conception intentionnelle du sens, qui subordonne ce dernier à l'étant, à l'être, à la vérité.

On peut partir de la comparaison entre Frege et Husserl. Le premier, dans "Sens et dénotation" caractérise le sens comme ce qui est partagé dans l'expression linguistique et à travers quoi le dénoté se présente, ou encore comme ce qui dans l'expression linguistique nous donne accès à ce dénoté. Le sens de Étoile du soir est cet accès à ou cette présentation de Vénus que fournit l'expression Étoile du soir. Diagrammatiquement: 


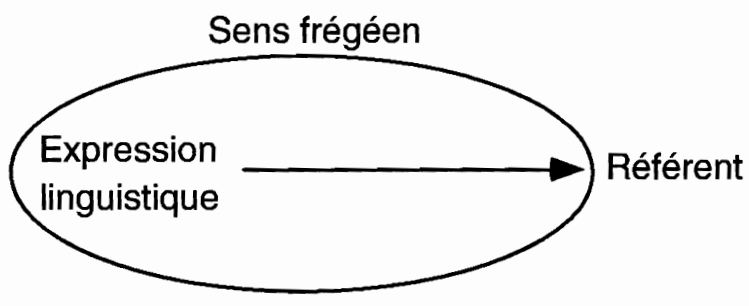

Le sens, c'est l'expression linguistique prise avec la flèche intentionnelle allant à l'objet dénoté, ou encore c'est l'expression linguistique transfigurée par cette flèche la faisant sortir d'elle-même pour aller à l'objet.

De même, dans Logique formelle et logique transcendantale, Husserl définit le sens comme "l'intentionné comme tel": le sens de pomme est, ainsi, la pomme en tant qu'elle est au bout d'une flèche partant de ma conscience qui la vise. Le sens, cette fois, c'est l'objet en tant que visé, c'est le contenu de dénotation transfiguré par le fait que je le rapporte à la flèche qui le vise, en quelque sorte. Diagrammatiquement:

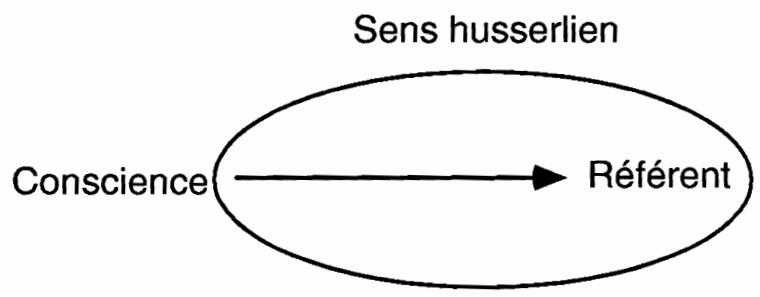

Les deux conceptions, donc, convergent profondément dans la détermination intentionnelle du sens, dans l'idée que le primordial quant au sens est qu'il conduit au référent: à ce qui passera ou vaudra pour l'existant, pour ce qui est, ce à l'égard de quoi le discours est capable de vérité. Pour Husserl la logique étudie le texte de la science pour en extraire l'intentionné comme tel (le sens), ce qui seul permet d'évaluer 


\section{JEAN-MICHEL SALANSKIS}

la vérité de ce texte scientifique. Pour Frege, le sens des expressions constituantes est ce qui, ultimement, permet la détermination de la valeur de vérité d'une phrase. Les deux conceptions nouent donc de manière extrêmement profonde et indéchirable la question philosophique du sens à celle de l'être et de la vérité, le concept de sens apparaissant comme un instrument au sein de la recherche qui concerne l'être et a la vérité comme enjeu.

Ajoutons que la postérité de nos auteurs ne modifie pas cette conception intentionnelle.

Les continuateurs de Husserl conservent le concept d'intentionnalité et accentuent même sa centralité. Le sens demeure pour eux essentiel, mais il se définit toujours comme la modalité de l'apparaitre de l'apparaissant, pour choisir une expression très usuelle aujourd'hui, où l'on retrouve littéralement ce que nous venons d'appeler conception intentionnelle du sens. Heidegger a ajouté à cette conception une étrange et fascinante inversion, qui en conserve néanmoins l'esprit, et qui le conduit à s'intéresser à la façon dont l'Être nous donne l'étant dans la déclosion: l'étant se trouve donc à nouveau pourvu d'un sens intentionnel, il est rapporté à la flèche qui aboutit à lui, mais cette flèche "provient" du fond sans mesure de l'Être au lieu de provenir de "nous". L'intentionnalité opère donc toujours et gouverne le concept de sens, mais cette intentionnalité vient du "dehors", du "fond", elle est, par rapport à nous, centripète plutôt que centrifuge.

Les continuateurs de Frege, pour une très grande partie d'entre eux, ont adhéré à l'idée que le sens des phrases s'égalait à l'ensemble de leurs conditions de vérité: comprendre une phrase, c'est comprendre selon quelle procédure rigoureuse et ordonnée je peux la vérifier (suivant la voie tracée par la sémantique de Tarski). En d'autres termes, le sens de la phrase réside dans la manière dont elle est par avance préparée à affronter le réel au cours d'une vérification: on n'a pas de peine 


\section{RESPONSABILI'IÉ SÉMANITQUE}

à retrouver dans cette définition la conception intentionnelle, c'est bien toujours par sa façon d'embrayer sur l'extérieur ontique que le linguistique est pris comme faisant sens.

Wittgenstein peut sembler avoir rompu avec cette conception en mettant en vedette, dans la conception du sens qu'on lui prête usuellement, la relation intersujets et le registre pratique. Le sens d'une expression s'égale à son emploi, diton lorsqu'on est wittgensteinien. Ou alors, on insiste sur le fait qu'il n'y a pas de langage privé, et que le sens est tributaire d'un comportement collectif, de ce qui a cours dans une forme de vie, dans un usage intersubjectif des mots. Mais si l'on regarde, par exemple, l'argument wittgensteinien sur l'impossibilité de spécifier en termes finis et dans le champ de l'intériorité les règles d'emploi des noms communs les plus ordinaires, comme table, on constate que la "solution sceptique" proposée au bout du compte, pour parler comme Kripke, consiste à se contenter d'une délimitation de l'extension référentielle de ces noms qui s'accomplit toute seule, aveuglément, dans le commerce linguistique et vital incluant ce mot avec les autres. Son argument, tout bien considéré, ne suspend pas la définition "intentionnelle" du sens, il dévoile seulement le mécanisme intersubjectif sur lequel la (les) flèche(s) intentionnelle(s) reposent.

\section{Ma compréhension du sens}

Le revirement que je suggère consiste tout simplement, pour le définir d'emblée, en le choix de comprendre le sens comme destinalité éprouvée. L'expérience primitive et directrice quant au sens est celle de la réception du sens, est celle où nous témoignons que nous avons été atteints, que nous avons reçu un message comme tel, c'est-à-dire comme s'adressant à nous. La flèche de la destination, celle qui va d'un destinateur - dont en l'occurrence la consistance ontologique ne joue 


\section{JEAN-MICHEL SALANSKIS}

aucun rôle, ne compte pas - à un destinataire, est ce qui fait critère ou ce autour de quoi se joue le faire sens, elle supplante donc la flèche intentionnelle dans cette nouvelle compréhension du sens.

Je fais observer, dans le livre, que déjà au niveau présignifiant de la réception d'un phonème, ce principe de destinalité joue: même une actualisation acoustique tombant au dehors de la région assignée aux variations acceptables d'un phonème compte comme émission de ce phonème si pour telle ou telle raison étrange elle a été acceptée comme lui à la réception ; inversement même une émission "légale", entrant dans le bassin de légitimité acoustique, manque à valoir comme émission du phonème si elle a été entendue de travers. L'émission d'un phonème est pour nous spontanément ce que j'appelle une transition étbique, quelque chose pour quoi le témoignage à l'arrivée fait critère.

J'ajoute qu'il en va de même avec la teneur sémantique des phrases. Quelle que soit la structure syntaxique et lexicale qu'on puisse identifier en elles par analyse de leur morphologie, la signification pour laquelle elles valent est toujours arrêtée en fonction d'un effet de contexte qui n'est pas seulement dépendant de la donne culturelle et de la situation d'énonciation, mais aussi des attentes, engagements et orientations du destinataire: celui-ci, en un sens, "choisit" toujours le sens au dernier moment en témoignant qu'il l'a reçu. Ce second point ayant été fort clairement et de façon très convaincante argumenté par T. Winograd et F. Florès, critiquant la première intelligence artificielle, après y avoir participé de façon éminente, dans Understanding Computers and Cognition. ${ }^{2}$

Je tire aussi de cette deuxième observation l'idée que la forme repérable que prend le message signifiant dans l'espace qui est le sien, forme que prétendent capter les arbres de la linguistique générative, ne contrôle donc pas le sens, pas plus que son émission, comme événement assignable dans le temps. 
Par rapport au temps, le destinal nous déconnecte de l'événement source, nous arrivons toujours au sens comme aux fresques des grottes de Lascault, comme à des vestiges d'un monde perdu avec lequel nous n'avons plus aucun lien, mais dont provient encore pour nous une atteinte. L'événement de notre réception signe le faire sens, mais ce n'est pas son individualité temporelle qui compte, elle peut se répéter ou se diviser en plusieurs épisodes, ce qui compte est, si j'ose dire, l'émotion du recevoir et pas sa qualité ou sa place temporelle.

L'idée de cette interprétation destinale du sens est ainsi que le sens doit être compris au-delà du temps et de l'espace, au-delà de l'être à vrai dire: il renvoie à l'intrigue éthique de l'atteinte du destinataire par le sens, il se comprend en termes d'une intrigue du sens analogue à l'intrigue éthique lévinasienne, selon laquelle le sujet athée apprend le sens de l'au-delà de l'être. Je propose dans le livre une description détaillée de cette intrigue du sens, en termes de trois moments: celui de l'enveloppement du sens, celui de la directionnalité du sens et celui du renvoi du sens.

L'enveloppement $d u$ sens désigne simplement ce qui distingue le sens de l'objet: le sens se présente à nous comme enveloppé en lui-même, en attente d'un déversement vers le destinataire; il a la profondeur d'un comprimé visant à s'exprimer, il charge ce qui le porte comme un excès. Alors que dans la notion d'objet figure l'idée d'une platitude et d'une suffisance, dans l'objet s'arrête et se maintient tout ce qui s'offre. Cet enveloppement du sens en lui-même fait que le sens me sollicite en s'exprimant.

La directionnalité $d u$ sens est ce qui est prescrit par le mot sens lui-mêtme, qui signifie volontiers la direction spatiale ou la direction temporelle (voir le panneau « sens unique » dans les rues des villles, ou le rêve d'inverser le sens du temps). Le sens met en jeu une direction, direction d'accomplissement du sens, 


\section{JEAN-MICHEL SALANSKIS}

que l'on peut traduire au plan existentiel comme la direction spatiale ou temporelle vers l'ailleurs ou le futur meilleurs.

Le renvoi du sens est la structure la plus banale articulant le signifiant et le signifié: chaque fois que nous enregistrons un faire sens, quelque chose a dû renvoyer à autre chose, le long de ce renvoi étant supposé s'accomplir l'explicitation du sens. L'attitude indéfiniment inquiète du philologue, toujours en attente d'un autre élément signifiant derrière celui qu'il lit, peut nous aider à concevoir dans sa généralité le renvoi du sens.

Au gré de la compréhension du sens que je propose, ces trois moments doivent être "entendus" dans la perspective de l'adresse et de la destinalité. Ce qui conduit aux réinterprétations suivantes:

L'enveloppement du sens se comprend comme sujet. En fin de compte, le destinataire impute toujours l'enveloppement du sens à un sujet de sens, construction ou hallucination seconde. Bien que l'intrigue du sens commence "par définition" dans l'ignorance du destinateur, de la consistance ontologique duquel on n'a nullement besoin, dont la position est superflue et indifférente, elle implique "en fin de compte" l'imputation de l'excès de ce qui me sollicite à un sujet de sens qui est le produit interprétatif de l'intrigue du sens. Lorsque je reçois le déchaînement de l'orage au dessus du lac comme à moi adressé, et que je détermine ainsi la relation comme réception sémantique, je ne peux faire autrement que camper un sujet de sens de cet orage, une "nature" allant au devant de mon état d'âme, typiquement.

La directionnalité du sens est toujours lue comme la directionnalité éthique fondamentale qui fait passer de moi à autrui, qui fait franchir l'abîme lévinasien évoqué plus haut, abîme dont le discours est pour Levinas l'unique passeur (qui respecte la distance en la franchissant). Tout se passe comme si la topologie discrète sur le doubleton \{moi, autrui\} surdéterminait toute les directionnalités possibles où le sens se laisse traduire. 


\section{RESPONSABILI'IÉ SÉMAN'ITQUE}

Le renvoi du sens est compris comme ayant sa motivation essentielle dans la demande. Si je passe du signifiant au signifié, c'est parce que c'est ce qui est demandé, je le sais dans ce saisissement par la sollicitation qui est l'expérience même du sens. Comprendre, c'est toujours comprendre comme ce qui est à comprendre le demande, comme il est demandé dans l'intrigue du sens reçu. La mesure fondamentale de l'herméneutique est éthique et pas ontique. Recevoir le sens, pour cette raison, c'est le relancer conformément à la demande: c'est cela qu'on appelle comprendre, et plus originairement, expliciter.

Ce dispositif de l'intrigue du sens une fois posé, mon livre s'efforce d'ajouter à la sobriété, la pauvreté volontaire de cette compréhension du sens ce qu'il faut pour accueillir, sans changer de perspective, toute la richesse de la variation culturelle du sens, tout le legs immense de la signification dans nos mondes, legs littéraire, scientifique, politique, religieux, juridique, etc. Je suis ainsi amené à décrire dans ses moments principaux la complexité du sens et à identifier ce que j'appelle les coordonnées du sens.

Je ne développerai pas ici ces points, renvoyant le lecteur qui en serait curieux à la source, c'est-à-dire, encore une fois, à mon ouvrage Sens et philosophie du sens.

Ce que je dois tenter de présenter maintenant néanmoins, c'est la façon dont je conçois cette "philosophie du sens" dont j'ai déjà annoncé que je la revendiquais, dans le même ouvrage et, depuis, dans l'ensemble de mon travail.

\section{Le projet de l'ethanalyse}

Dans le livre, j'explique qu'une philosophie du sens doit 1) prendre son départ dans le sens, se déployer à partir de lui (exigence générale) ; 2) comprendre le sens sans le réduire à 
autre chose, comme un primitif (exigence de radicalité) ; 3) s'atteler à la description de tout l'horizon humain du sens, dans sa richesse et sa diversité (exigence programmatique). L'ethanalyse, mot forgé par croisement de éthique et de psychanalyse, est l'accomplissement de ce troisième volet programmatique. Je prend acte de la régionalité du sens: il y a des régions du sens, chacune d'entre elles correspond à une demande qui transite le long des circuits de l'adresse entre les hommes, chaque nouvel acteur recevant le sens en question comme exigeant d'être relancé conformément à ce qu'il demande. Chaque région est "supportée" par un ethos, une coutume humaine supposée relayer et faire valoir le sens en cause, coutume qui, en général, organise la récurrence de contenus de conscience, d'esquisses linguistiques et de pratiques. Je suggère ainsi qu'il y a un ethos de l'amour: il y a une tradition de l'amour, nous recevons l'amour comme un sens à illustrer à nouveau, à satisfaire, c'est-à-dire à relancer en entendant les prescriptions de l'amour, en les obéissant, les interprétant et les répercutant tout à la fois. La tâche de l'ethanalyse est de dégager les éléments directeurs qui caractérisent ce qui vaut comme relance adéquate de l'amour, de décrire le faisceau de prescriptions qui donne à l'amour son identité de sens (j'appelle ce faisceau de prescriptions sémance de l'amour, analogue de l'essence dans la phénoménologie décalée qu'est l'ethanalyse). Une phénoménologie classique connaît les régions comme régions d'objets, et cherche l'essence caractérisant la donnée de tels objets. Mes régions sont des régions de sens, je soutiens en particulier que le "champ" de l'amour n'est pas le champ des objets amoureux, il n'y a pas de tels objets à caractériser vis-àvis d'autres objets possibles, l'amour est plutôt un sens de comportement, ou, disons, d'épisode humain, d'affaire humaine: c'est pourquoi l'ethanalyse va chercher à expliciter la sémance de l'amour comme un système d'exigences qui sont 


\section{RESPONSABILI'IÉ SÉMAN'ITQUE}

ce qui demande à être compris en demandant à être activement satisfait dans l'amour. La région s'annonce en général par le biais d'un sollicitant (ici, le mot amour, jeté dans notre culture). L'ethanalyse pratique une sorte de réduction phénomenologique, très difficile à décrite dans ce qui en serait la voie générale, dont le principe consiste à tenter de revenir mentalement à un monde indemne du sens en cause: à reconstruire et retrouver la demande qui transite dans l'amour en concevant que le jeu de l'amour puisse être abandonné, non relancé. Technique qui doit nous adresser aux circonstances directrices dont dépend qu'il n'en aille pas ainsi.

Jusqu'à présent, j'ai suivi le programme de l'ethanalyse à propos de l'amour et du politique, qui me semblaient de bons exemples de sollicitants, mais les travaux correspondants ne sont pas publiés. J'ai, de plus, publié une ethanalyse du fait juif. ${ }^{3}$ Le cas m'est apparu comme très favorable, parce que, assez clairement pour beaucoup je crois, nous sommes embarrassés avec le mot juif, bien, tout en sachant que l'adjectif juif ne synthétise pas un ensemble de propriétés ontiques, vérifiables dans le réel et permettant la spécification d'une classe d'objets ou d'individus. Que juifse laisse plus proprement saisir comme un sollicitant apparaît ainsi comme particulièrement plausible.

Une question que l'on peut poser naturellement, après avoir entendu l'ensemble de ma présentation de la philosophie du sens, est celle de la retombée possible d'une telle philosophie sur le langage et la question du langage.

D'un côté, il ne faut pas attendre que la philosophie du sens se substitue à la philosophie du langage pour apporter exactement les mêmes fruits qu'elle, il ne faut donc pas exiger de la philosophie du sens une focalisation sur le langage et une mise en vedette du langagier analogues. Si j'ai souhaité, à partir d'une inspiration lévinasienne, le déplacement vers une philosophie du sens, c'est notamment parce que je ne me 


\section{JEAN-MICHEL SALANSKIS}

satisfaisais pas de la primitivité souvent accordée au langage. Je ne parviens pas à comprendre le geste de "reconduction au langage" qui est si souvent celui de la philosophie contemporaine autrement que comme la reconduction à une couche d'être privilégiée (quand cette reconduction n'équivaut pas, plus simplement et comme il était expliqué plus haut, à la subordination de toute question philosophique à la question et à l'horizon de la vérité). De toute façon, il me semble que la philosophie du langage accentue le langage au sein d'une dispute ontologique et en vue de l'être en quelque sorte. Certes, il resterait l'option de prendre le langage comme hors être ou comme ce dans l'élément de quoi se comprend et s'annonce le bors être. Mais de ce point de vue, je suis convaincu par Levinas que seul l'intervalle éthique nous enseigne le hors-être.

Cela étant, l'affaire du langage est passionnante, et la philosophie est tellement compromise avec le langage que ne pas réfléchir sur le langage serait un programme absurde, qui ne me tente nullement. Donc, le projet que je définis et que je défends commande une certaine forme de vigilance philosophique à l'égard du langage.

Pour une part, de ce point de vue, je marche sur les traces des recherches contemporaines qui interrogent le langage de manière phénoménologique. Dans mon dernier ouvrage Herméneutique et cognition, ${ }^{4}$ je commente longuement les travaux de R. Langacker, F. Rastier, ${ }^{5}$ en montrant combien et comment leur entreprise de description non grammaticale, non computationnelle de la signification linguistique rejoint les topos majeurs de la phénoménologie et de l'herméneutique. Depuis, l'ouvrage de P. Cadiot et Y.-M. Visetti, Pour une théorie des formes sémantiques ${ }^{6}$ est paru, qui va encore plus loin dans ce sens, et qui expose en particulier le sémantisme lexical en termes de l'Être-au-monde merleau-pontien de façon très convaincante. Ces différentes recherches me semblent toucher juste scientifiquement, et, de plus, préparer le terrain à un exa- 
men de la signification linguistique dans l'horizon de l'adresse, dans la mesure où elles mettent en relief l'attitude qui est derrière le vouloir dire, ce qui offre un cadre pour la prise en vue de la "condition éthique" du sens.

Dans une version non publiée de Sens et philosophie du sens, j'allais un peu plus loin en essayant de repérer, dans la manifestation linguistique elle-même, des éléments qui signaleraient, agiraient ou expliciteraient à leur manière la relativité du sens à l'adresse, ou plus essentiellement qui dévoileraient la dimension de la demande derrière le renvoyer du sens. J'avais alors cru devoir tenter une analyse de deux tours de la rhétorique du français, le en tant que, qui est tout à fait international ${ }^{7}$, et le il en est de, qui l'est peut être moins, mais que Levinas avait relevé lui aussi, pour en donner une interprétation "ontologiste" si ma mémoire est juste.

Je vais maintenant, pour finir cette intervention, revenir à Levinas, afin de juger rétrospectivement du rapport de ce qui précède avec sa pensée, avant d'aborder, à cette occasion, un nouvel enjeu de la philosophie contemporaine.

\section{Retour à Levinas: la question du fondement}

Dans l'ouvrage Sens et philosophie du sens lui-même, avant d'introduire ma conception de l'intrigue du sens, résumée plus haut, je me réfère à un texte de Levinas où celui-ci semble traiter de la question du sens comme telle.

Dans L'bumanisme de l'autre homme, en effet, Levinas enregistre le fait que, pour la phénoménologie contemporaine, lc scns est pluricl ct dessinc par cettc pluralité toutc la modulation culturelle du monde. Le monde n'existe qu'en tant que corrélat intentionnel, et donc nous le connaissons suivant un sens, au sens de la conception intentionnelle du sens qui est celle de la phénoménologie. La projection intentionnelle étant 
ouverte à tous et en tout mode, les "modulations du monde" se juxtaposent et se superposent en liberté.

Levinas redoute alors que, dans le contexte de cette fragmentation, chaque sens en vienne à se signifier lui-mêtme plutôt que quoi que ce soit d'autre que soi. Que le jeu intentionnel du sens résorbe le sens dans l'affirmation de cette parcelle de vue auto-suffisante que chacun porte. Alors qu'après tout, la conception intentionnelle du sens veut elle aussi que le sens soit lié à une vraie flèche, s'adressant à un extérieur.

Il soutient donc qu'il doit y avoir un proto-sens, un sens de tous les sens qui part de moi ou de l'humanité pour aller vraiment au-delà de soi, de tout soi. Un sens qui va au-delà du monde, à Dieu si l'on accepte de prendre la formule comme dans une certaine mesure définitoire de ce qu'on entend par Dieu. Ce sens est pour lui notamment celui de l'CEurre, qu'il entend comme l'œuvre se détachant de nous mais que nous cherchons à accomplir par delà l'effet de retour pour nous, que nous valorisons par delà notre propre mort à la limite. En sorte que ce proto-sens ou sens unique qui porte tous les sens parait accompli par le discours dans la valeur que lui donne Totalité et infini, et que nous rappelions au début de cette intervention: le discours comme ce qui a la faculté de se porter vers l'incommensurable ou l'irréductible sans le ramener à soi, même s'il le catégorise.

Par rapport à ce que dit Levinas dans ce texte, et que je présente dans le livre, bien évidemment, comme ma source d'inspiration, on peut, je crois, discerner dans ma construction quelque chose comme un renversement tendanciel: alors que Levinas thématise le sens d'abord comme centrifuge, comme partant de moi pour aller vers autrui, pour aller vraiment au-delà de moi, alors que, donc, il lie le sens à un franchissement de l'intervalle éthique lié à la topologie discrète originaire sur \{moi, autrui\} de la forme moi-->autrui, je "saisis" plutôt le directeur et le fondamental du sens au niveau de la 
réception moi<--(...): dans ceci que quelqu'un (moi) témoigne de son atteinte par ce qu'il peut aussitôt décrire comme une requête du sens $((\ldots))$. Le (...), ici, est un comme si autrui, que le moi destinataire va aussitôt construire ou halluciner comme sujet.

Il faudrait relativiser cette distinction, parce que Levinas peut décrire parfois les choses de la manière qui vient d'être dite aussi. Mais il me semble qu'il n'a pas tendance à le faire lorsqu'il présente le sens dans sa généralité: c'est plutôt pour décrire la "condition d'otage" qui est au cœur de l'éthique qu'il insiste sur la situation du destinataire, de manière même finalement hyperbolique.

Cette différence quasi-formelle correspond à mon avis à ceci que, chez Levinas, le niveau du sens n'est pas séparé de manière volontariste de celui de l'éthique. Levinas tend spontanément à décrire l'irruption de l'éthique comme l'irruption du sens même, ou réciproquement. Mon livre au contraire, et c'est même le point qui dicte à cette intervention son titre, s'attache à distinguer responsabilité sémantique et responsabilité éthique.

Je le fais notamment de manière extrêmement simple en prenant l'exemple de la question «Pouvez-vous porter cette valise?». ${ }^{8}$ Il est possible de répondre simplement «Oui» à cette question, qui, pourtant, n'est visiblement pas une question s'insérant dans un débat théorique portant sur ce dont je suis capable, mais une demande déguisée dans la question. Cette réponse traîtresse sera néanmoins un acte de responsabilité sémantique, elle correspond à une manière de prendre la phrase comme m'ayant atteint, et de répondre suivant une interprétation de ce qu'elle demande. Mais elle tombe loin de la responsabilité éthique à l'égard du prochain dont parle Levinas.

Cet exemple témoigne de ce que, dans ma description de "l'intrigue du sens », je produis une sorte de plan minimal du 
sens, que l'on peut voir comme celui d'une éthique pauvre ou indigente, nous vouant tous à la relance du sens reçu sans nous vouer au visage. Je crois en effet à l'importance de ce niveau du sens, où ne sommes déjà plus dans la neutralité de l'être, puisque nous sommes en situation de réception et que nous avons à charge, constamment, de témoigner de la nonindifférence du reçu en le lisant comme demande, mais où nous ne sommes pas encore dans le concernement éthique authentique.

Le dernier point qui m'importe est de tenter d'élucider en termes de fondement la relation qui s'établit entre mon plan du sens et le plan de l'éthique lévinasienne.

Un aspect de cette relation est d'ores et déjà évident: ma conception de l'intrigue du sens se fonde sur celle de l'intrigue éthique selon Levinas. Ce que je soutiens, fort clairement, est que pour comprendre dans son authenticité le faire sens, nous devons concevoir le destinataire comme affecté par le message indépendamment de tout "secours" ontologique, comme entendant la pure demande (de relance, de renvoi) constitutive de l'atteinte du sens. La pensée du sens selon l'adresse est donc complètement tributaire de l'idée lévinasienne de la réquisition éthique, de l'être-en-dette du sujet enjoint. Reconnaissons donc que la pensée lévinasienne de l'éthique est ici en position de fondement.

Mais n'interprétons pas, en même temps, cette relation de fondation en termes ontologiques, ce qui est rendu tentant par la grande faveur, dans la discussion intraphénoménologique connue de moi, de l'idée ontologique du fondement: lorsque quelque chose est allégué comme fondement, pour beaucoup d'oreilles philosophiques, cela veut dire que ce quelque chose aurait dans son être la ressource d'engendrer ce qu'il fonde, cela veut dire qu'il est pris comme principe ontologique, à partir duquel une dérivation au sens de la genèse a lieu. Bien entendu, déclarer l'éthique lévinasienne 
comme fondatrice à l'égard de mon "plan du sens" selon un tel modèle ne conviendrait pas du tout, cela irait à l'encontre du mode philosophique que Levinas nous suggère. Lorsqu'il présente en général l'éthique comme philosophie première, cela n'est pas, et de toute nécessité si on l'a compris, en un tel sens: l'éthique n'est pas la raison d'être de l'être, elle n'est pas une archè dont l'être et ce qui a trait à l'être se laisseraient engendrer, prenant à revers la quête de l'originaire qui est celle du courant ontologisant dominant de la tradition philosophique grecque.

Nous tâcherons plutôt de comprendre que la relation éthique de Levinas est un prérequis dans l'ordre du sens pour la compréhension de l'intrigue du sens: c'est donc suivant la dimension an-ontologique du sens elle-même que l'éthique fonde le sens, et pas du tout en termes de dérivation ontologique, de genèse. Et ce qui est dit à cet endroit peut à mon avis être généralisé: lorsque Levinas donne l'éthique comme la "nouvelle" philosophie première, nous devons compléter en explicitant ceci que la "dérivation" de l'ensemble des strates extra-éthiques, ontologiques eu premier chef, à partir de l'an-ontologique de l'éthique, est à interpréter comme transition dans la dimension elle-même an-ontologique du sens. Ainsi, lorsque Levinas régresse à partir de l'expérience du tiers vers le droit, la justice, le langage, la logique, il nous fait voir le "problème éthique" comme originaire par rapport à tous ces termes selon le sens. le droit, la justice, le langage et la logique sont situés dans la spécificité de sens qui est la leur par cette fonction qu'ils ont de répondre à la demande qui émane de la situation à trois.

J'achèverai le propos de cette intervention en tirant de ce qui précède une conclusion quant à la question ou l'enjeu du fondement dans le contexte philosophique contemporain. Comme on le sait, de nombreuses approches, au cours du vingtième siècle, ont convergé pour "dénoncer" ou 
"disqualifier" le projet philosophique du fondement, de Nietzsche au Quine de Naturalized Epistemology. Un très large consensus a rassemblé des philosophes d'obédiences variées, qui s'accordaient pour réfuter la prétention qui avait pu être celle de la philosophie de rattacher toutes les performances de la culture à des racines qu'elle serait seule à savoir nommer, et dont elle saurait, unique en cela à nouveau, expliquer la relation fondatrice à ce qu'elles fondent.

Levinas nous offre une voie pour sauver quelque chose de cet anti-fondationnalisme tout en ne sacrifiant pas le travail séculaire de la philosophie sur la légitimation. Ce travail témoigne en effet de ce que, en philosophie, on s'interroge non seulement sur l'ordre dans lequel peuvent être exposées et articulées les vérités ou les concepts, les vérités se déduisant de vérités primitives et les concepts se définissant en termes de concepts primitifs, mais aussi, plus profondément, sur la chaîne de renvois du sens qui fait comparaittre telle déclinaison du sens après telle autre plus primitive. Cette interrogation est par excellence le propre de l'attitude philosophante en tant que la philosophie, qu'elle le sache ou nom, est depuis toujours en charge de la dimension du sens et de l'ordonnancement qu'elle abrite. Inversement, s'interroger à ce niveau est corrélatif d'un abandon radical et absolu de la prétention à dire la hiérarchie ontologique organisant toutes choses selon des rapports de genèse: la philosophie résolument fondationnelle dans la dimension du sens est tout aussi résolument anti-fondationnaliste selon l'acception ontologique, c'est-à-dire métaphysique du fondement.

Voilà pourquoi, après Levinas, j'ai le sentiment de suivre une voie encore moins fondationnaliste que les philosophies contemporaines, tout en revendiquant contre elles une forme de fondationnalisme, aussi nécessaire à la philosophie, à mes yeux, que l'eau à l'être humain abandonné dans le désert. 


\section{RESPONSABILI'IÉ SÉMAN'ITQUE}

\section{Notes}

${ }^{1}$ Jean-Michel Salanskis, Sens et philosophie du sens (Paris, Desclée de Brouwer, 2001).

2 Terry Winograd and Fernando Florès, Understanding Computers and Cognition: $A$ New Foundation for Design (Ablex, Norwood, N.J.: Ablex, 1986).

${ }^{3}$ Jean-Michel Salanskis, Extermination, loi, Israël: Ethanalyse du fait juif (Paris: Les Belles Lettres, 2003).

${ }^{4}$ Jean-Michel Salanskis, Herméneutique et cognition (Lille: Presses du Septentrion, 2003).

${ }^{5}$ Ronald W. Langacker, Foundations of Cognitive Grammar I: Theoretical Prerequisites, and Foundations of Cognitive Grammar II: Descriptive Application (Stanford: Stanford University Press, 1999); and François Rastier, Sémantique interprétative (Paris: PUF, 1996).

${ }^{6}$ Pierre Cadiot and Yves-Marie Visetti, Pour une théorie des formes sémantiques (Paris, PUF, 2001).

${ }^{7} \mathrm{~A}$ ma connaissance, il a justifié un livre de philosophie analytique, qui cherchait à formaliser la contribution du qua aux phrases.

${ }^{8}$ Reprenant un exemple qu'utilisait J.-F. Lyotard dans son séminaire, si mon souvenir est exact. 